\title{
The space-clamped Hodgkin-Huxley system with random synaptic input: inhibition of spiking by weak noise and analysis with moment equations
}

\author{
Henry C. Tuckwell ${ }^{1,2}$ and Susanne Ditlevsen ${ }^{3}$ \\ tuckwell@adelaide.edu.au \\ ${ }^{1}$ School of Electrical and Electronic Engineering, University of Adelaide \\ SA 5005, Australia \\ ${ }^{2}$ School of Mathematical Sciences, Monash University \\ Clayton, Victoria 3168, Australia \\ ${ }^{3}$ Department of Mathematical Sciences, University of Copenhagen \\ DK 2100 Copenhagen $\phi$, Denmark \\ Email addresses \\ tuckwell@adelaide.edu.au; susanne@math.ku.dk
}

July 27, 2021

\begin{abstract}
We consider a classical space-clamped Hodgkin-Huxley model neuron stimulated by synaptic excitation and inhibition with conductances represented by OrnsteinUhlenbeck processes. Using numerical solutions of the stochastic model system obtained by an Euler method, it is found that with excitation only there is a critical value of the steady state excitatory conductance for repetitive spiking without noise and for values of the conductance near the critical value small noise has a powerfully inhibitory effect. For a given level of inhibition there is also a critical value of the steady state excitatory conductance for repetitive firing and it is demonstrated that noise either in the excitatory or inhibitory processes or both can powerfully inhibit spiking. Furthermore, near the critical value, inverse stochastic resonance was observed when noise was present only in the inhibitory input process.
\end{abstract}


The system of 27 coupled deterministic differential equations for the approximate first and second order moments of the 6-dimensional model is derived. The moment differential equations are solved using Runge-Kutta methods and the solutions are compared with the results obtained by simulation for various sets of parameters including some with conductances obtained by experiment on pyramidal cells of rat prefrontal cortex. The mean and variance obtained from simulation are in good agreement when there is spiking induced by strong stimulation and relatively small noise or when the voltage is fluctuating at subthreshold levels. In the occasional spike mode sometimes exhibited by spinal motoneurons and cortical pyramidal cells the assunptions underlying the moment equation approach are not satisfied. 


\section{Contents}

1 Introduction

2 The $\mathrm{HH}$ equations with random synaptic currents 4 2.1 Description of model . . . . . . . . . . . . . . . . . 5

3 Simulation results $\quad 7$

3.1 Simulations and the inhibitory effect of noise . . . . . . . . . . . . 7

3.1.1 Excitation: critical value and choice of $\Delta t \ldots \ldots \ldots \ldots$

3.1.2 Inhibition by noise with excitation only . . . . . . . . . . . 8

3.1.3 Inhibition by noise with excitation and inhibition . . . . . . . . . 9

4 Differential equations for the approximate first and second order moments $\quad \mathbf{1 1}$

4.1 The means . . . . . . . . . . . . . . . . . . . . 12

4.2 The variances . . . . . . . . . . . . . . . . . . . . . . . . . . . . . . . . . . . . . .

4.3 Remaining covariances . . . . . . . . . . . . . . . 16

5 Comparison of results for moment equations and simulation $\quad \mathbf{1 7}$

5.1 Examples where moment equations and simulation give good agreement . . . 17

5.1 .1 Occasional spike mode . . . . . . . . . . . . . . . 18

5.1.2 Input parameters of Destexhe et al. (2001) . . . . . . . . . . . . 19

6 Discussion and conclusions $\quad 23$

7 Appendix: HH parameters, coefficients and their derivatives 25

\section{Introduction}

The stochastic nature of neuronal discharges was first reported in the 1930s, through experiments which found variability in the responses of frog myelinated axon to shocks of the same intensity and duration, notably by Monnier and Jasper (1932), Blair and Erlanger (1932) and Pecher $(1936,1937)$. Concerning the last mentioned author, Verveen has documented an interesting account of his career, which started in Belgium and ended mysteriously at the age of 28 in the United States after researching radioactive substances whose nature was considered a military secret. (See www.verveen.eu which also has links to Pecher's articles (in French)). Some notable later contributions, including review, on this aspect of stochasticity in neurons are Verveen (1960), Lecar and Nossal (1971) and Clay (2005).

Since these early discoveries, there have been a very large number of experimentalist studies which revealed the stochastic nature of nearly all neuronal activity, the latter term referring mainly to action potential generation. This embraces single neurons and their component parts and populations of neurons and glia. Some of the pioneering works were 
on interspike interval variability in muscle spindles (Brink et al., 1946), cells in the auditory system (Gerstein and Kiang, 1960) and those in the visual cortex (Burns and Webb, 1976).

Mathematical modeling of the complex dynamical processes underlying such activity has since flourished (Bachar et al., 2013). Much of the theoretical work has focused on channel noise at both the single channel level (Colquhoun and Hawkes, 1981) and at the patch level modeled by a diffusion limit for a birth and death process (Tuckwell, 1987). The role of such noise in the generation of action potentials, for example by altering the firing threshold, has been explored in (for example) White et al. (2000), Austin (2008) and Li et al. (2010).

Linear models with synaptic noise have been studied since the 1960s and there have been more recent analyses of them such as those of Hillenbrand (2002), Ditlevsen (2007) and Berg and Ditlevsen (2013). Most nonlinear models of neuronal activity are based on HodgkinHuxley (1952) type systems and include spatial models (Horikawa, 1991; Sauer and Stannat, 2016). Some recent studies include those of Wenning et al. (2005), who employed a model similar to that explored in the present article, and Finke et al. (2008), who included both additive noise and channel noise in the form of Ornstein-Uhlenbeck processes (OUPs) for some activation variables, and Yi et al. (2015), who studied noise effects on spike threshold in a two-dimensional Hodgkin-Huxley $(\mathrm{HH})$ type model with synaptic noise represented by an OUP.

The focus of the present article is on the classical HH system with synaptic noise and its analysis by the moment method, in a similar vein to that for the same system with additive white noise (Rodriguez and Tuckwell, 2000; Tuckwell and Jost, 2009). With weak additive white noise or with conductance-based noise as in the present model (Tuckwell et al., 2009) or with colored noise (Guo, 2011), and mean input currents near the threshold for repetitive firing, the firing rate undergoes a minimum (inverse stochastic resonance) as the noise level increases from zero. We examine the responses of the system to synaptic input, either excitatory alone, or with inhibition near the threshold for repetitive spiking and compare solutions obtained by the moment method with simulation. The moment method and extensions of it has been employed in several recent studies of neuronal networks (Deco and Marti, 2007; Mareiros et al., 2009; Hasegawa, 2009; Deco et al., 2013; Franović et al., 2013; Hasegawa, 2015) and genetic networks (Sokolowski and Tkačik, 2015).

\section{The HH equations with random synaptic currents}

The standard $\mathrm{HH}$ system consists of four ordinary differential equations for the electrical potential $V(t)$, the potassium activation variable $n(t)$, the sodium activation variable $m(t)$ and the sodium inactivation variable $h(t)$. The latter three variables take values in $[0,1]$ and their differential equations involve the coefficients $\alpha_{n}, \beta_{n}, \alpha_{m}, \beta_{m}, \alpha_{h}, \beta_{h}$ which depend on $V$. In a previous article (Tuckwell and Jost, 2009), the HH system with an additive current including deterministic and random components was analyzed. Here we use similar techniques for the $\mathrm{HH}$ system with random synaptic excitation and inhibition. See also Tuckwell and Jost (2012) and Bashkirtsiva et al. (2015) for further analysis of the effects of noise in the $\mathrm{HH}$ model with additive noise.

Random synaptic inputs can be included in several ways (e.g., Rodriguez and Tuckwell, 
1998), but we restrict our attention to cases where there are no discontinuities in $V$ but rather the synaptic currents can be represented with diffusion processes, which makes the mathematical formalism somewhat less complicated. The resultant system consists of 6 coupled stochastic differential equations (SDEs) where the stochasticity is only explicit in the 5th and 6th equations.

The form of the systems under consideration here is that of an $n$-dimensional temporally homogeneous diffusion process $\mathbf{X}=\{\mathbf{X}(t), t \geq 0\}$ where the $j$-th component satisfies the stochastic differential equation

$$
d X_{j}(t)=f_{j}(\mathbf{X}(t)) d t+\sum_{k=1}^{m} g_{j k}(\mathbf{X}(t)) d W_{k}(t)
$$

where the $W_{k}$ s are independent standard (zero mean, variance $t$ at time $t$ ) Wiener processes and it is assumed that conditions for existence and uniqueness of solutions are met (Gihman and Skorohod, 1972).

\subsection{Description of model}

For the present model we let the first four variables be $X_{1}=V, X_{2}=n, X_{3}=m$ and $X_{4}=h$ and $\mathbf{X}$ be the vector of all 6 components. The first equation of a general $\mathrm{HH}$ system with synaptic inputs can be written

$$
d X_{1}=\frac{1}{C}\left(H(\mathbf{X})+I_{s y n}\right) d t
$$

where $I_{\text {syn }}$ represents the synaptic input currents and we have defined the sum of the standard $\mathrm{HH}$ currents as

$$
H(\mathbf{X})=\bar{g}_{K} X_{2}^{4}\left(V_{K}-X_{1}\right)+\bar{g}_{N a} X_{3}^{3} X_{4}\left(V_{N a}-X_{1}\right)+g_{L}\left(V_{L}-X_{1}\right)
$$

where $\bar{g}_{K}, \bar{g}_{N a}$ and $g_{L}$ are the maximal (constant) potassium, sodium and leak conductances per unit area with corresponding equilibrium potentials $V_{K}, V_{N a}$, and $V_{L}$, respectively. The 2nd, 3rd and 4th equations take the standard $\mathrm{HH}$ forms,

$$
\begin{aligned}
d X_{2}=f_{2}(\mathbf{X}) d t=\left[\alpha_{n}\left(X_{1}\right)\left(1-X_{2}\right)-\beta_{n}\left(X_{1}\right) X_{2}\right] d t \\
d X_{3}=f_{3}(\mathbf{X}) d t=\left[\alpha_{m}\left(X_{1}\right)\left(1-X_{3}\right)-\beta_{m}\left(X_{1}\right) X_{3}\right] d t \\
d X_{4}=f_{4}(\mathbf{X}) d t=\left[\alpha_{h}\left(X_{1}\right)\left(1-X_{4}\right)-\beta_{h}\left(X_{1}\right) X_{4}\right] d t
\end{aligned}
$$

In the following we denote the derivatives of $\alpha_{n}$ etc. by $\alpha_{n}^{\prime}$ etc.

A representation of certain synaptic inputs has been successfully used in models for the spontaneous random spiking of cat and rat neocortical pyramidal neurons (Destexhe et al., 2001; Fellous et al., 2003) in which the excitatory and inhibitory inputs constituting the ongoing background input are presumed to be composed mainly of small and frequent glutamatergic and GABA-ergic postsynaptic currents which could be separately identified. A similar model was employed for an $\mathrm{HH}$ neuron with conductance-driven input by Tuckwell 
et al. (2009) in a demonstration of the robustness of the phenomenon of inverse stochastic resonance. In the Destexhe et al. (2001) model there is, in addition to the usual three $\mathrm{HH}$ currents, an M-type potassium current which, as in Tuckwell et al. (2009), is not included here. In actual neocortical pyramidal cells there are several more component currents than in the Destexhe et al. (2001) model - see for example Yu et al. (2008) for a partial list. The overall synaptic current is then written by Destexhe et al. (2001) as

$$
I_{\text {syn }}=\frac{1}{A}\left[g_{e}(t)\left(V_{E}-V\right)+g_{i}(t)\left(V_{I}-V\right)\right],
$$

where $V_{E}, V_{I}$ are equilibrium potentials for excitatory and inhibitory synaptic input, the synaptic excitatory and inhibitory conductances at time $t$ are $g_{e}(t)$ and $g_{i}(t)$, respectively, and $A$ is the "total membrane area". The synaptic conductances are ascribed the stochastic differential equations

$$
\begin{aligned}
d g_{e}(t) & =-\frac{1}{\tau_{e}}\left(g_{e}(t)-\bar{g}_{e}\right) d t+\sigma_{e} d W_{e}(t) \\
d g_{i}(t) & =-\frac{1}{\tau_{i}}\left(g_{i}(t)-\bar{g}_{i}\right) d t+\sigma_{i} d W_{i}(t)
\end{aligned}
$$

where $\tau_{e}, \tau_{i}$ are time constants, $\bar{g}_{e}, \bar{g}_{i}$ are equilibrium values, $W_{e}, W_{i}$ are corresponding (assumed independent) standard Wiener processes and $\sigma_{e}, \sigma_{i}$ are noise amplitudes. Thus the processes $g_{e}$ and $g_{i}$ are of the Ornstein-Uhlenbeck type.

In this model, the first of the 6 SDEs is simply

$$
d X_{1}=f_{1}(\mathbf{X}) d t
$$

with

$$
f_{1}(\mathbf{X})=\frac{1}{C}\left(H(\mathbf{X})+\frac{X_{5}}{A}\left(V_{E}-X_{1}\right)+\frac{X_{6}}{A}\left(V_{I}-X_{1}\right)\right)
$$

where $X_{5}=g_{e}$ and $X_{6}=g_{i}$.

For $X_{5}$ and $X_{6}$ we have

$$
d X_{5}=f_{5}\left(X_{5}\right) d t+g_{51} d W_{1}
$$

and

$$
d X_{6}=f_{6}\left(X_{6}\right) d t+g_{62} d W_{2}
$$

where we identify $W_{e}=W_{1}$ and $W_{i}=W_{2}$, and where

$$
\begin{aligned}
& f_{5}\left(X_{5}\right)=-\frac{1}{\tau_{e}}\left(X_{5}-\bar{g}_{e}\right) \\
& f_{6}\left(X_{6}\right)=-\frac{1}{\tau_{i}}\left(X_{6}-\bar{g}_{i}\right) .
\end{aligned}
$$

Further $g_{51}=\sigma_{e}$ and $g_{62}=\sigma_{i}$ and these are the only two non-zero $g_{j k} \mathrm{~s}$ in this model so $m=2$ in Equ. (1). There are 27 distinct first and second order moments for this model and differential equations for their approximations are obtained in Section 3. 


\section{Simulation results}

\subsection{Simulations and the inhibitory effect of noise}

Simulated solutions of the system of 6 coupled SDEs equations defining the above $\mathrm{HH}$ model neuron with random synaptic inputs are obtained with a simple Euler method in which discretization is applied with a time step of $\Delta t$. Unless stated otherwise, the value $A=1$ is employed in the calculations described below.

\subsubsection{Excitation: critical value and choice of $\Delta t$}

With no inhibition and no noise so that $\sigma_{e}=0$, there is a critical value of $\bar{g}_{e}$ for repetitive firing. When $\bar{g}_{e}=0.1$ repetitive (presumed continuing indefinitely) firing does not occur for any value of $\Delta t$ tested. Figure $1 \mathrm{~A}$ shows the voltage response without noise and with $\Delta t=0.002$. There is only one spike at about $t \approx 5$. With very small noise $\sigma_{e}=0.0005$, the results are shown in Figure 1B for 10 trials, the response being almost identical in each trial. When the noise amplitude is greater at $\sigma_{e}=0.005$ as in Figure $1 \mathrm{C}$, second spikes may emerge as is the case in 4 of the ten trials depicted. Figure 1D shows 4 voltage trajectories for a still greater noise level $\sigma_{e}=0.05$. Here there are in all cases (many not shown) an apparently unceasing sequence of randomly occurring spikes, despite the fact that the value of $\bar{g}_{e}$ is less than the critical value for repetitive firing without noise.

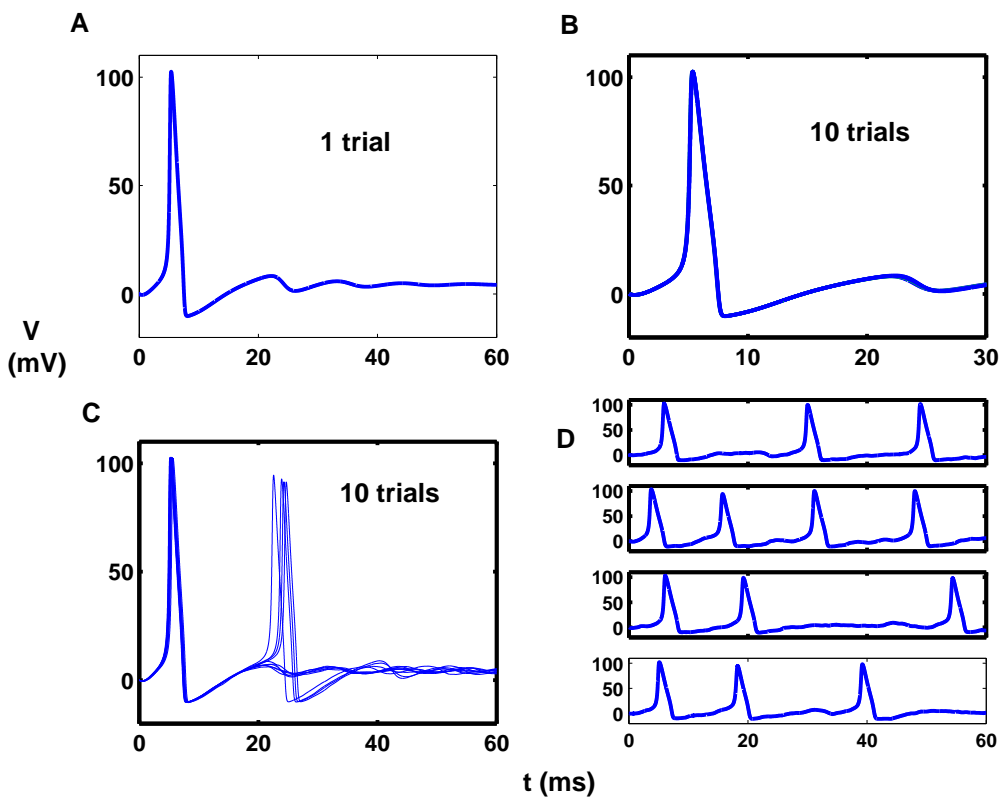

Figure 1: Simulation results with excitation only and various noise levels. In all cases steady state excitation $\bar{g}_{e}=0.10$ below the critical value for repetitive firing and a time step of $\Delta t=0.002$. A. No noise $\left(1\right.$ trial) giving one spike. B. Ten trials with noise $\sigma_{e}=0.0005$. One spike only, being practically the same on each trial. C. Ten trials with $\sigma_{e}=0.005$. The noise is sufficient to give rise to a second spike in 4 of the ten trials. D. 4 individual trials with $\sigma_{e}=0.05$. In all cases examined multiple sustained spiking at random times occurs. 
Using the previously found (Tuckwell et al., 2009) critical value $\bar{g}_{e}=0.112$ as a guide, spike trains were examined for values of $\bar{g}_{e}$ close to that value for various values of $\Delta t$. Results will be reported only for the two values $\Delta t=0.015$ and $\Delta t=0.002$. For $\bar{g}_{e}=0.111$, there were 4 and 6 spikes respectively for the smaller and larger time steps, and for the slightly larger value $\bar{g}_{e}=0.1115$ there were 4 spikes of declining amplitude for the smaller time step whereas for the larger time step there were 13 spikes in $240 \mathrm{~ms}$ whose amplitudes finally remained constant - see Figure 2A. With $\bar{g}_{e}=0.112$ there were 7 spikes of declining amplitude for the smaller time step and an apparently repetitive train for the larger time step.

Finally, with $\bar{g}_{e}=0.1125$ just above the previously determined critical value an apparently stable repetitive spike train was obtained with both the larger and smaller time step. This is seen in Figure 2 and suggests that the critical value is very close to 0.1125 . In general it was observed that the larger time step sped up the spiking and tended to make it more stable. However, the above results indicated that it is preferable to use the smaller time step despite its leading to significantly greater computation times.

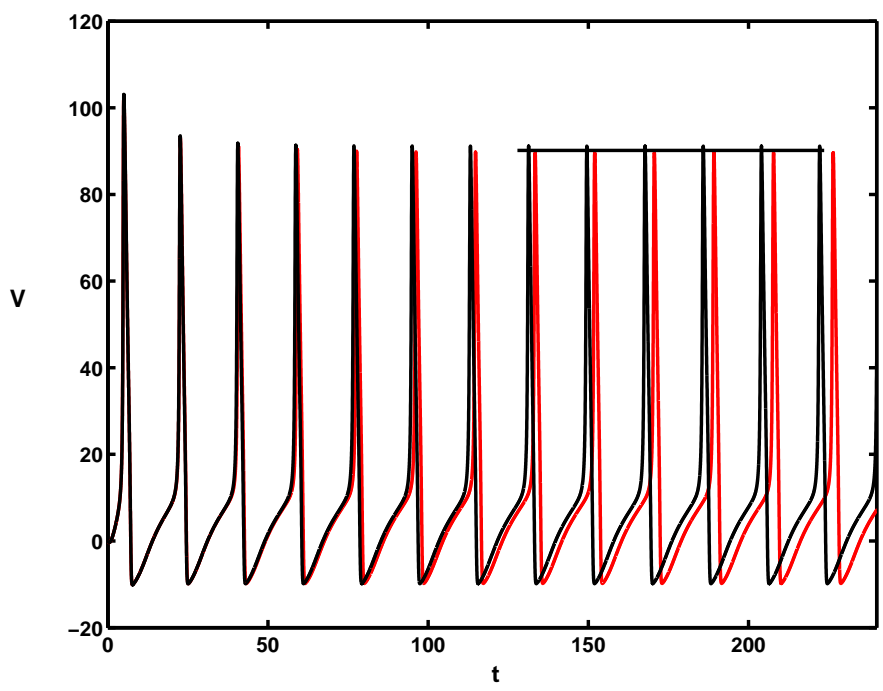

Figure 2: Spike trains for $\bar{g}_{e}=0.1125$ just above the critical value for repetitive spiking, showing the effects of smaller (red) and larger (black) time steps of 0.002 and 0.015 respectively. The larger time step leads to a higher frequency. Horizontal segment indicates stable spike amplitudes for both time steps.

\subsubsection{Inhibition by noise with excitation only}

With excitation only at $\bar{g}_{e}=0.1125$, so that without noise there is repetitive periodic firing, noise of a small amplitude can lead to a greatly reduced number of spikes. This is illustrated in Figure 3 where the three columns show 4 trials, of length $100 \mathrm{~ms}$, for each of three values of $\sigma_{e}$, being $0.0025,0.01$ and 0.025 , increasing from left to right. With no noise there are 6 spikes (see Figure 2). The smallest of the values of $\sigma_{e}$ leads to the greatest reduction in 
average spike numbers, to 3.0, whereas the largest value of $\sigma_{e}$ has an average spike number of 5.75 .
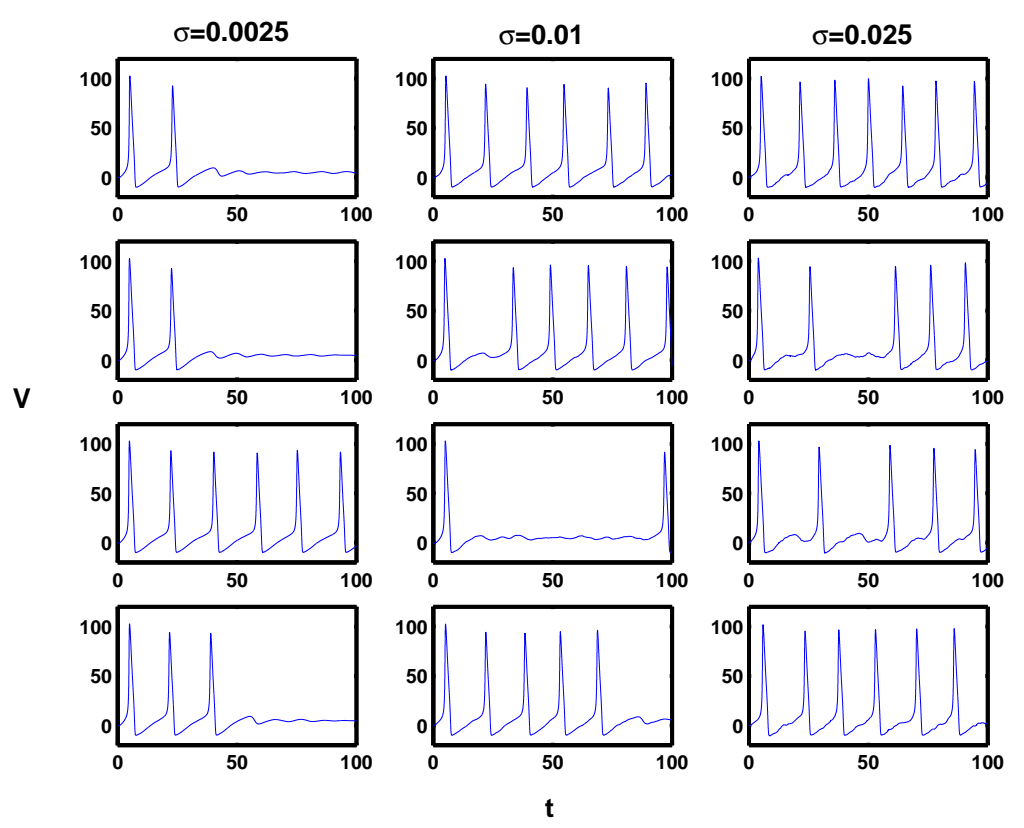

Figure 3: Spike trains for $\bar{g}_{e}=0.1125$ just above the critical value for repetitive spiking, showing the effects of small noise of three different magnitudes, increasing from left to right, in 4 trials.

\subsubsection{Inhibition by noise with excitation and inhibition}

In previous investigations of the inhibitory effects of noise on repetitive firing induced by synaptic input (Tuckwell et al., 2009), only excitatory inputs have been considered. Here we briefly consider a few cases in which the synaptic input is both excitatory and inhibitory and there is noise in either the excitatory or inhibitory component or both. First it was required to find a combination of excitation and inhibition which would lead to repetitive spiking in the deterministic case. Without a formal proof, it seems that for any level of inhibition $\bar{g}_{i}$ there can always be found a level of excitation $\bar{g}_{e}$ to give repetitive spiking. With $\bar{g}_{i}=0.1125$, close to the critical value of $\bar{g}_{e}$ for excitation only, spike trains were examined with a time step of 0.015 for various values of $\bar{g}_{e}$. The elicited trains for $\bar{g}_{e}=0.1725$ and $\bar{g}_{e}=0.1775$ did not exhibit repetitive spiking as shown in the first two panels in the top row of Figure 4. When $\bar{g}_{e}$ was increased slightly to 0.1790 , as in the right hand panel of the first row, repetitive spiking was sustained, indicating that this level of excitation was critical for the given level of inhibition. In the remaining three rows of Figure 4 are shown the resulting spike trains with noise for three trials in which the values of $\bar{g}_{e}$ and $\bar{g}_{i}$ are those which gave repetitive spiking without noise. In the second row there is noise of a small magnitude $\sigma_{e}=0.0025$ in the excitation only, whereas in the third row noise of magnitude $\sigma_{i}=0.0025$ is present in the inhibitory input only. Finally, in the 4th row there is noise in both the 
excitatory and inhibitory input processes with $\sigma_{e}=\sigma_{i}=0.00125$ such that the sum of the amplitudes is the same as in rows two and three. The percentage reductions in average spike numbers, for these (small) sample sizes, are 78,67 and 64 for noise in excitation, inhibition and both, respectively. This preliminary investigation indicates that inhibition of repetitive spiking by noise is as strong when there is inhibition present as when there is excitation only and occurs regardless of whether the noise arises in excitatory, inhibitory or both input processes.
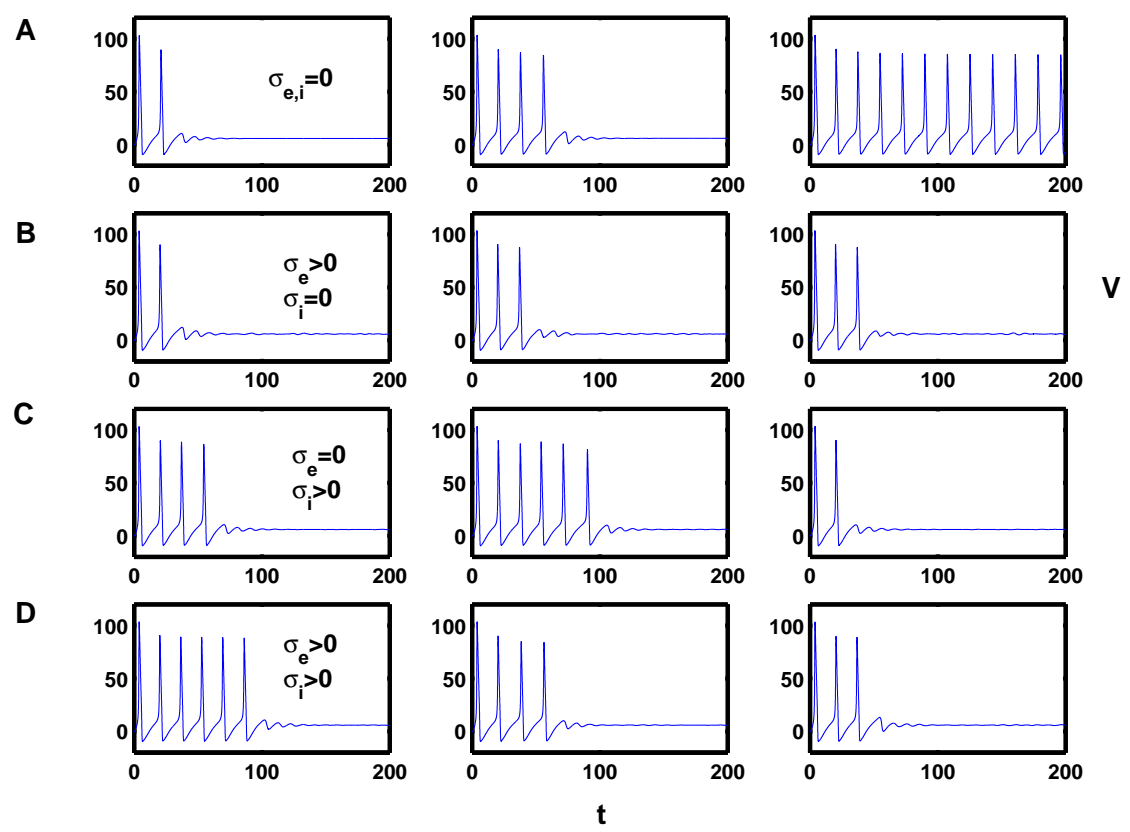

Figure 4: A. In the top three records there is no noise but both excitatory and inhibitory inputs. In the left-most panel, with $\bar{g}_{e}=0.1725$ and $\bar{g}_{i}=0.1125$, and in the middle panel with $\bar{g}_{e}=0.1775$ and $\bar{g}_{i}=0.1125$, repetitive firing is not established. When, as in the righthand panel, the larger value $\bar{g}_{e}=0.1790$ is employed with the same value of $\bar{g}_{i}$, repetitive firing occurs. B. In the results of the second row the values of $\bar{g}_{e}$ and $\bar{g}_{i}$ are as in the right-hand panel of the first row, leading to repetitive spiking in the absence of noise, but that now a small noise $\sigma_{e}=0.0025$ is added only to the excitatory component, giving rise to a large degree of inhibition. C. As in B but that now noise with amplitude $\sigma_{i}=0.0025$ is added to only the inhibitory component, which also inhibits the spiking. D. As in B and $\mathrm{C}$ except that the noise is spread equallly amongst excitatory and inhibitory inputs with $\sigma_{e}=0.00125$ and $\sigma_{i}=0.00125$. Significant inhibition of spiking is observed in this case also.

Further results were obtained with the levels of excitation and inhibition $\bar{g}_{e}$ and $\bar{g}_{i}$ which led to repetitive spiking as in Figure 4A. 50 trials of length 100 ms were performed for 30 values of the inhibitory noise parameter $\sigma_{i}$, from 0 to 0.1 , with no noise in the excitatory process. The mean number of spikes versus noise level is shown in Figure 5, black circles, and it is seen that the spike rate undergoes a minimum around values of $\sigma_{i}$ just less than 0.02. Away from the critical value for repetitive spiking, when the level of the 
excitatory input was increased to $\bar{g}_{e}=0.2106$ but with the same value of $\bar{g}_{i}$, the firing rate undergoes a much weaker minimum as $\sigma_{i}$ is increased from 0 to 0.1 . These results parallel those obtained previously for increasing the excitatory noise level (Tuckwell et al., 2009). Somewhat surprisingly, therefore, the phenomenon of inverse stochastic resonance occurs with increasing level of inhibitory noise alone.

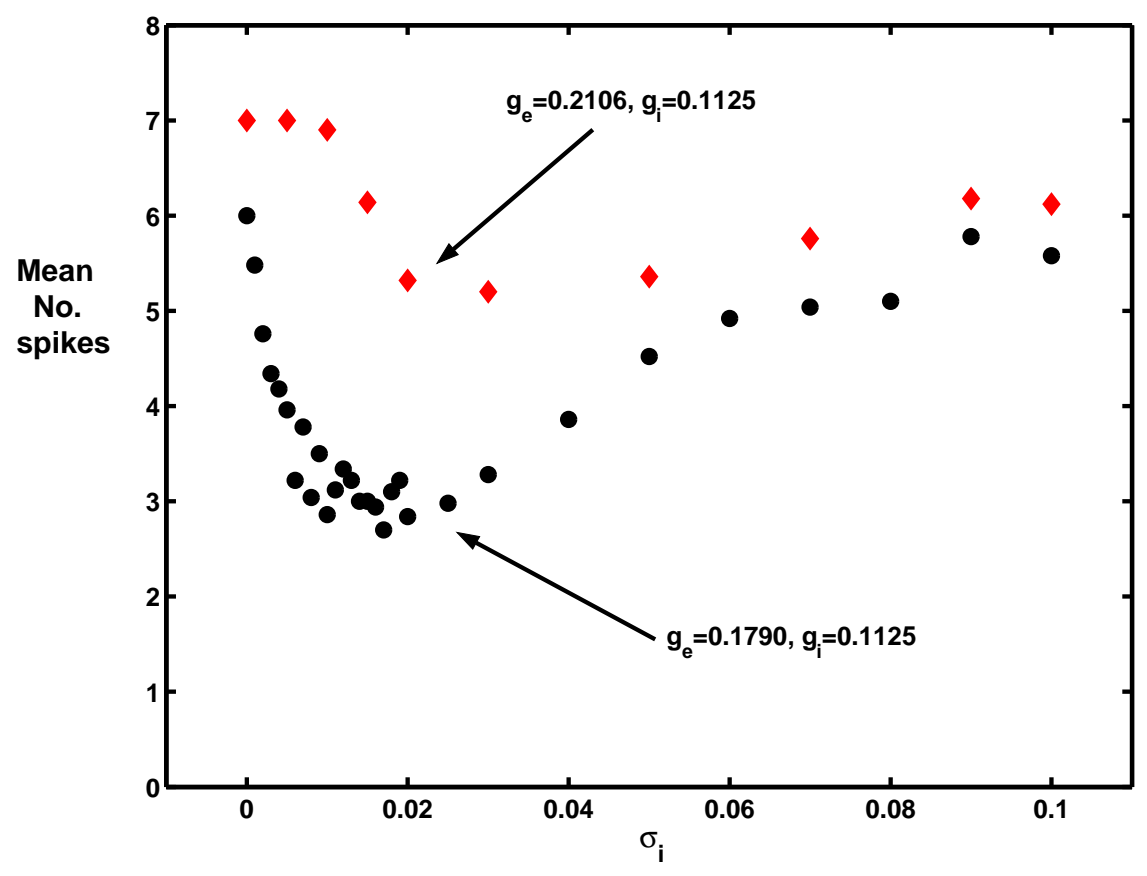

Figure 5: The mean number of spikes per trial is plotted against the inhibitory noise parameter $\sigma_{i}$, with no noise in the excitatory process, for values of $\bar{g}_{e}$ and $\bar{g}_{i}$ near a critical value for repetitive spiking (black circles) and at a level of excitation above the critical value (red diamonds). For the first set a pronounced minimum occurs in the firing rate near $\sigma=0.02$, providing evidence of inverse stochastic resonance with respect to inhibitory noise alone.

\section{Differential equations for the approximate first and second order moments}

We will find deterministic differential equations satisfied by the approximations for the means, variances and covariances of the components of the $n$-component vector-valued random process $\mathbf{X}$ in the above model, using the scheme of Rodriguez and Tuckwell (1996). Accordingly, for relatively small noise amplitudes the exact means $\mu_{i}(t)=E\left[X_{i}(t)\right]$ and covariances $K_{i j}(t)=E\left[\left(X_{i}(t)-\mu_{i}(t)\right)\left(X_{j}(t)-\mu_{j}(t)\right)\right]$ may sometimes be approximated by the functions $m_{i}(t), i=1, \ldots, n$, and $C_{i j}(t), i, j=1, \ldots, n$, respectively, which obey a system of deterministic differential equations. 
The vector of means at time $t$ is denoted by $\mathbf{m}(t)$. These quantities are found to satisfy the following systems of ordinary differential equations

$$
\frac{d m_{i}}{d t}=f_{i}(\mathbf{m})+\frac{1}{2} \sum_{l=1}^{n} \sum_{p=1}^{n}\left\{\frac{\partial^{2} f_{i}}{\partial x_{l} \partial x_{p}}\right\} C_{(\mathbf{m})} C_{l p}
$$

whereas the covariances are determined by

$$
\begin{aligned}
\frac{d C_{i j}}{d t}= & \sum_{k=1}^{n}\left\{g_{i k} g_{j k}\right\}_{(\mathbf{m}, t)}+\sum_{l=1}^{n}\left\{\frac{\partial f_{i}}{\partial x_{l}}\right\}_{(\mathbf{m}, t)} C_{l j}+\sum_{l=1}^{n}\left\{\frac{\partial f_{j}}{\partial x_{l}}\right\}_{(\mathbf{m}, t)} C_{i l} \\
& +\frac{1}{2} \sum_{k=1}^{n} \sum_{l=1}^{n} \sum_{p=1}^{n}\left\{g_{j k} \frac{\partial^{2} g_{i k}}{\partial x_{l} \partial x_{p}}+\frac{\partial g_{i k}}{\partial x_{l}} \frac{\partial g_{j k}}{\partial x_{p}}+\frac{\partial g_{i k}}{\partial x_{p}} \frac{\partial g_{j k}}{\partial x_{l}}+g_{i k} \frac{\partial^{2} g_{j k}}{\partial x_{l} \partial x_{p}}\right\}_{(\mathbf{m}, t)} C_{l p} .
\end{aligned}
$$

In the present model this equation is simpler because the last line of Equ. (17) is absent, there being no triple sum contribution involving second derivatives or products of first order derivatives of the $g_{j k}$ s because such terms are all constants, most of which are zero.

In order to simplify the notation in the following equations we give some definitions. Let

$$
H_{1}(\mathbf{m})=\bar{g}_{K} m_{2}^{4}+\bar{g}_{N a} m_{3}^{3} m_{4}+g_{L}+\frac{m_{5}}{A}+\frac{m_{6}}{A}
$$

and let

$$
\begin{aligned}
H_{2}\left(\mathbf{m}, y_{1}, y_{2}, y_{3}, y_{4}, y_{5}\right)= & \frac{4}{C} \bar{g}_{K} m_{2}^{3}\left(V_{K}-m_{1}\right) y_{1}+\frac{3}{C} \bar{g}_{N a} m_{3}^{2} m_{4}\left(V_{N a}-m_{1}\right) y_{2} \\
& +\frac{1}{C} \bar{g}_{N a} m_{3}^{3}\left(V_{N a}-m_{1}\right) y_{3}+\frac{1}{A C}\left[\left(V_{E}-m_{1}\right) y_{4}+\left(V_{I}-m_{1}\right) y_{5}\right] .
\end{aligned}
$$

\subsection{The means}

Evaluating the required first and second order partial derivatives of $f_{1}, . ., f_{6}$ we obtain the following differential equations for the means $m_{1}, \ldots, m_{6}$, of $V, n, m, h, g_{i}, g_{e}$, respectively. For the voltage,

$$
\begin{aligned}
\frac{d m_{1}}{d t}= & \frac{1}{C}\left[H(\mathbf{m})+\frac{m_{5}}{A}\left(V_{E}-m_{1}\right)+\frac{m_{6}}{A}\left(V_{I}-m_{1}\right)\right. \\
& -4 \bar{g}_{K} m_{2}^{3} C_{12}-3 \bar{g}_{N a} m_{3}^{2} m_{4} C_{13}-\bar{g}_{N a} m_{3}^{3} C_{14}-\frac{C_{15}}{A}-\frac{C_{16}}{A} \\
& +6 \bar{g}_{K} m_{2}^{2}\left(V_{K}-m_{1}\right) C_{22}+3 \bar{g}_{N a} m_{3} m_{4}\left(V_{N a}-m_{1}\right) C_{33} \\
& \left.+3 \bar{g}_{N a} m_{3}^{2}\left(V_{N a}-m_{1}\right) C_{34}\right]
\end{aligned}
$$


For the auxiliary variables

$$
\begin{aligned}
\frac{d m_{2}}{d t}= & \alpha_{n}\left(m_{1}\right)\left(1-m_{2}\right)-\beta_{n}\left(m_{1}\right) m_{2}+\frac{1}{2}\left(\alpha_{n}^{\prime \prime}\left(m_{1}\right)\left(1-m_{2}\right)-\beta_{n}^{\prime \prime}\left(m_{1}\right) m_{2}\right) C_{11} \\
& -\left(\alpha_{n}^{\prime}\left(m_{1}\right)+\beta_{n}^{\prime}\left(m_{1}\right)\right) C_{12} \\
\frac{d m_{3}}{d t}= & \alpha_{m}\left(m_{1}\right)\left(1-m_{3}\right)-\beta_{m}\left(m_{1}\right) m_{3}+\frac{1}{2}\left(\alpha_{m}^{\prime \prime}\left(m_{1}\right)\left(1-m_{3}\right)-\beta_{m}^{\prime \prime}\left(m_{1}\right) m_{3}\right) C_{11} \\
& -\left(\alpha_{m}^{\prime}\left(m_{1}\right)+\beta_{m}^{\prime}\left(m_{1}\right)\right) C_{13} \\
\frac{d m_{4}}{d t}= & \alpha_{h}\left(m_{1}\right)\left(1-m_{4}\right)-\beta_{h}\left(m_{1}\right) m_{4}+\frac{1}{2}\left(\alpha_{h}^{\prime \prime}\left(m_{1}\right)\left(1-m_{4}\right)-\beta_{h}^{\prime \prime}\left(m_{1}\right) m_{4}\right) C_{11} \\
& -\left(\alpha_{h}^{\prime}\left(m_{1}\right)+\beta_{h}^{\prime}\left(m_{1}\right)\right) C_{14} .
\end{aligned}
$$

For the means of the synaptic conductances we have

$$
\frac{d m_{5}}{d t}=-\frac{1}{\tau_{e}}\left(m_{5}-\bar{g}_{e}\right), \quad \frac{d m_{6}}{d t}=-\frac{1}{\tau_{i}}\left(m_{6}-\bar{g}_{i}\right) .
$$

Since these are means of OUPs, exact solutions are known, which in fact coincide with the approximations. Solutions are

$$
m_{5}(t)=m_{5}(0) e^{t / \tau_{e}}+\bar{g}_{e}\left(1-e^{t / \tau_{e}}\right)
$$

and likewise for $m_{6}(t)$.

\subsection{The variances}

For the above model of an $\mathrm{HH}$ system with random synaptic input, the differential equations for the variances of $X_{i}(t)$, obtained by substituting the appropriate derivatives and coefficients are as follows. For the variance of $V$ we have

$$
\begin{aligned}
\frac{d C_{11}}{d t}= & -\frac{2}{C} H_{1}(\mathbf{m}) C_{11}+\frac{8}{C} \bar{g}_{K} m_{2}^{3}\left(V_{K}-m_{1}\right) C_{12}+\frac{6}{C} \bar{g}_{N a} m_{3}^{2} m_{4}\left(V_{N a}-m_{1}\right) C_{13} \\
& +\frac{2}{C} \bar{g}_{N a} m_{3}^{3}\left(V_{N a}-m_{1}\right) C_{14}+\frac{2}{A C}\left[\left(V_{E}-m_{1}\right) C_{15}+\left(V_{I}-m_{1}\right) C_{16}\right] .
\end{aligned}
$$

For the variances of $n, m$ and $h$ we find

$$
\begin{aligned}
& \frac{d C_{22}}{d t}=2\left[\left(\alpha_{n}^{\prime}\left(m_{1}\right)\left(1-m_{2}\right)-\beta_{n}^{\prime}\left(m_{1}\right) m_{2}\right) C_{12}-\left(\alpha_{n}\left(m_{1}\right)+\beta_{n}\left(m_{1}\right)\right) C_{22}\right] \\
& \frac{d C_{33}}{d t}=2\left[\left(\alpha_{m}^{\prime}\left(m_{1}\right)\left(1-m_{3}\right)-\beta_{m}^{\prime}\left(m_{1}\right) m_{3}\right) C_{13}-\left(\alpha_{m}\left(m_{1}\right)+\beta_{m}\left(m_{1}\right)\right) C_{33}\right] \\
& \frac{d C_{44}}{d t}=2\left[\left(\alpha_{h}^{\prime}\left(m_{1}\right)\left(1-m_{4}\right)-\beta_{h}^{\prime}\left(m_{1}\right) m_{4}\right) C_{14}-\left(\alpha_{h}\left(m_{1}\right)+\beta_{h}\left(m_{1}\right)\right) C_{44}\right]
\end{aligned}
$$

For the variances of the excitatory and inhibitory conductances we find

$$
\frac{d C_{55}}{d t}=\sigma_{e}^{2}-\frac{2}{\tau_{e}} C_{55}, \quad \frac{d C_{66}}{d t}=\sigma_{i}^{2}-\frac{2}{\tau_{i}} C_{66} .
$$


Since these variances are for OUPs we know the exact solutions

$$
C_{55}(t)=\frac{\sigma_{e}^{2} \tau_{e}}{2}\left(1-e^{2 t / \tau_{e}}\right)
$$

and likewise for $C_{66}(t)$, coinciding with the approximations. 



\subsection{Remaining covariances}

The remaining 15 covariances are solutions of the following differential equations.

$$
\begin{aligned}
& \frac{d C_{12}}{d t}=\left[\alpha_{n}^{\prime}\left(m_{1}\right)\left(1-m_{2}\right)-\beta_{n}^{\prime}\left(m_{1}\right) m_{2}\right] C_{11}-\left[\alpha_{n}\left(m_{1}\right)+\beta_{n}\left(m_{1}\right)\right] C_{12} \\
& -\frac{1}{C} H_{1}(\mathbf{m}) C_{12}+H_{2}\left(\mathbf{m}, C_{22}, C_{23}, C_{24}, C_{25}, C_{26}\right) \\
& \frac{d C_{13}}{d t}=\left[\alpha_{m}^{\prime}\left(m_{1}\right)\left(1-m_{3}\right)-\beta_{m}^{\prime}\left(m_{1}\right) m_{3}\right] C_{11}-\left[\alpha_{m}\left(m_{1}\right)+\beta_{m}\left(m_{1}\right)\right] C_{13} \\
& -\frac{1}{C} H_{1}(\mathbf{m}) C_{13}+H_{2}\left(\mathbf{m}, C_{23}, C_{33}, C_{34}, C_{35}, C_{36}\right) \\
& \frac{d C_{14}}{d t}=\left[\alpha_{h}^{\prime}\left(m_{1}\right)\left(1-m_{4}\right)-\beta_{h}^{\prime}\left(m_{1}\right) m_{4}\right] C_{11}-\left[\alpha_{h}\left(m_{1}\right)+\beta_{h}\left(m_{1}\right)\right] C_{14} \\
& -\frac{1}{C} H_{1}(\mathbf{m}) C_{14}+H_{2}\left(\mathbf{m}, C_{24}, C_{34}, C_{44}, C_{45}, C_{46}\right) \\
& \frac{d C_{15}}{d t}=-\frac{1}{C} H_{1}(\mathbf{m}) C_{15}+H_{2}\left(\mathbf{m}, C_{25}, C_{35}, C_{45}, C_{55}, C_{56}\right)-\frac{1}{\tau_{e}} C_{15} \\
& \frac{d C_{16}}{d t}=-\frac{1}{C} H_{1}(\mathbf{m}) C_{16} H_{2}\left(\mathbf{m}, C_{26}, C_{36}, C_{46}, C_{56}, C_{66}\right)-\frac{1}{\tau_{i}} C_{16} \\
& \frac{d C_{23}}{d t}=\left(\alpha_{m}^{\prime}\left(m_{1}\right)\left(1-m_{3}\right)-\beta_{m}^{\prime}\left(m_{1}\right) m_{3}\right) C_{12}+\left(\alpha_{n}^{\prime}\left(m_{1}\right)\left(1-m_{2}\right)-\beta_{n}^{\prime}\left(m_{1}\right) m_{2}\right) C_{13} \\
& -\left(\alpha_{m}\left(m_{1}\right)+\beta_{m}\left(m_{1}\right)+\alpha_{n}\left(m_{1}\right)+\beta_{n}\left(m_{1}\right)\right) C_{23} \\
& \frac{d C_{24}}{d t}=\left(\alpha_{h}^{\prime}\left(m_{1}\right)\left(1-m_{4}\right)-\beta_{h}^{\prime}\left(m_{1}\right) m_{4}\right) C_{12}+\left(\alpha_{n}^{\prime}\left(m_{1}\right)\left(1-m_{2}\right)-\beta_{n}^{\prime}\left(m_{1}\right) m_{2}\right) C_{14} \\
& -\left(\alpha_{n}\left(m_{1}\right)+\beta_{n}\left(m_{1}\right)+\alpha_{h}\left(m_{1}\right)+\beta_{h}\left(m_{1}\right)\right) C_{24} \\
& \frac{d C_{25}}{d t}=\left(\alpha_{n}^{\prime}\left(m_{1}\right)\left(1-m_{2}\right)-\beta_{n}^{\prime}\left(m_{1}\right) m_{2}\right) C_{15}-\left(\alpha_{n}\left(m_{1}\right)+\beta_{n}\left(m_{1}\right)\right) C_{25}-\frac{1}{\tau_{e}} C_{25} \\
& \frac{d C_{26}}{d t}=\left(\alpha_{n}^{\prime}\left(m_{1}\right)\left(1-m_{2}\right)-\beta_{n}^{\prime}\left(m_{1}\right) m_{2}\right) C_{16}-\left(\alpha_{n}\left(m_{1}\right)+\beta_{n}\left(m_{1}\right)\right) C_{26}-\frac{1}{\tau_{i}} C_{26} \\
& \frac{d C_{34}}{d t}=\left(\alpha_{h}^{\prime}\left(m_{1}\right)\left(1-m_{4}\right)-\beta_{h}^{\prime}\left(m_{1}\right) m_{4}\right) C_{13}+\left(\alpha_{m}^{\prime}\left(m_{1}\right)\left(1-m_{3}\right)-\beta_{m}^{\prime}\left(m_{1}\right) m_{3}\right) C_{14} \\
& -\left(\alpha_{m}\left(m_{1}\right)+\beta_{m}\left(m_{1}\right)+\alpha_{h}\left(m_{1}\right)+\beta_{h}\left(m_{1}\right)\right) C_{34} \\
& \frac{d C_{35}}{d t}=\left(\alpha_{m}^{\prime}\left(m_{1}\right)\left(1-m_{3}\right)-\beta_{m}^{\prime}\left(m_{1}\right) m_{3}\right) C_{15}-\left(\alpha_{m}\left(m_{1}\right)+\beta_{m}\left(m_{1}\right)\right) C_{35}-\frac{1}{\tau_{e}} C_{35} \\
& \frac{d C_{36}}{d t}=\left(\alpha_{m}^{\prime}\left(m_{1}\right)\left(1-m_{3}\right)-\beta_{m}^{\prime}\left(m_{1}\right) m_{3}\right) C_{16}-\left(\alpha_{m}\left(m_{1}\right)+\beta_{m}\left(m_{1}\right)\right) C_{36}-\frac{1}{\tau_{i}} C_{36} \\
& \frac{d C_{45}}{d t}=\left(\alpha_{h}^{\prime}\left(m_{1}\right)\left(1-m_{4}\right)-\beta_{h}^{\prime}\left(m_{1}\right) m_{4}\right) C_{15}-\left(\alpha_{h}\left(m_{1}\right)+\beta_{h}\left(m_{1}\right)\right) C_{45}-\frac{1}{\tau_{e}} C_{45} \\
& \frac{d C_{46}}{d t}=\left(\alpha_{h}^{\prime}\left(m_{1}\right)\left(1-m_{4}\right)-\beta_{h}^{\prime}\left(m_{1}\right) m_{4}\right) C_{16}-\left(\alpha_{h}\left(m_{1}\right)+\beta_{h}\left(m_{1}\right)\right) C_{46}-\frac{1}{\tau_{i}} C_{46}
\end{aligned}
$$


However, the correct solution of the last equation must be $C_{56}=0$ since the Wiener processes $W_{e}$ and $W_{i}$ are independent. Hence the terms in $H_{2}$ in the equations for $C_{15}$ and $C_{16}$ become $H_{2}\left(\mathbf{m}, C_{25}, C_{35}, C_{45}, C_{55}, 0\right)$ and $H_{2}\left(\mathbf{m}, C_{26}, C_{36}, C_{46}, 0, C_{66}\right)$.

\section{Comparison of results for moment equations and simulation}

\subsection{Examples where moment equations and simulation give good agreement}

Generally agreement between the results for the moment equation method (MEM) and simulation, in the sense that the mean and variance of the voltage as functions of time were reasonably close, was obtained when the net mean driving force was large and the variances small. This is to be expected from the assumptions under which the moment equations are derived.

Figure 6 shows one such case where there is excitation only with parameter values for synaptic input, $\bar{g}_{e}=3, \sigma_{e}=0.0003, \tau_{e}=2, V_{E}=80,50$ trials and standard Hodgkin-Huxley parameters as given in the appendix.

For most input parameter sets examined the maximum variance for the MEM was greater than that obtained by simulation. In a very few examples, particularly for large $\bar{g}_{e}$ and with a very small time step, the maximum variance for the simulation solution was greater than that for the MEM. The approximation for the variance could be overestimating the true variance, but it could also reflect that either there is a very small probability that the first spike would be delayed, advanced or even entirely inhibited leading to an increased variance, or that the amplitude in rare cases is larger during the first spike, also increasing the variance, but these probabilities are so small that they are not seen in practice in the simulations.

In a second example with reasonable agreement for the two methods there is synaptic excitation and synaptic inhibition with parameters $\bar{g}_{e}=3, \bar{g}_{i}=1, \sigma_{e}=0.0003, \sigma_{i}=0.0002$, $\tau_{e}=2, \tau_{i}=6, V_{E}=80, V_{I}=-10$ and standard $\mathrm{HH}$ parameters as given in the appendix. 30 simulation runs of 50 trials each were performed. Again, the mean of $V(t)$ obtained by each method was almost identical, as seen in the top part of Figure 7, where there is one blue curve for the MEM and 30 red curves for the simulations.

The corresponding results for the variance of $V(t)$ are shown in the bottom part of Figure 7. For the MEM the maximum variance of $V(t)$ is 0.0015 which is greater than the maximum variance in each of the 30 simulation runs. For the latter, the maximum value of all the maxima is 0.0014, which is fairly close to the value for the MEM, and the minimum value is 0.00069 , with an average of 0.0010 , which is about $33 \%$ less than the MEM value. For 29 of the 30 runs, simulation values of $\operatorname{Var}[V(t)]$ were less than the MEM value for all $t$ and in the remaining case the simulation value crossed the MEM value just after the peak value and remained above it for most of the falling phase. 

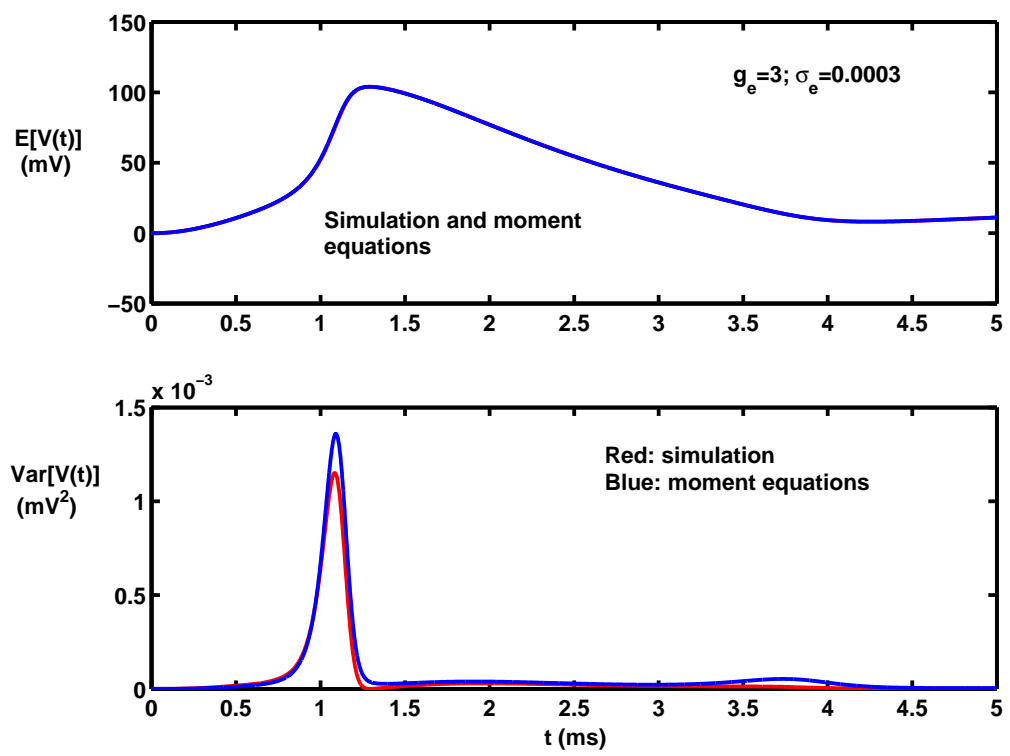

Figure 6: An example, with excitation only, of the calculated mean and variance of the voltage $V(t)$ as functions of time of the voltage where there is good agreement between results for simulation (red) and for moment equations (blue). The input parameters are $\bar{g}_{e}=3$ and $\sigma_{e}=0.0003$, with no inhibition and standard HH parameters. The means for the two methods are indistinguishable.

\subsubsection{Occasional spike mode}

The spiking reported in Destexhe et al. (2001) and Fellous et al. (2003) is classified as being from a cell operating in the occasional spike mode (Calvin, 1975), a term first used with reference to spinal motoneuron spiking. There is insufficient net depolarizing current to give rise to a sustained train of action potentials but occasional large excursions to supra-threshold states arise due to random synaptic input.

With a small depolarizing current of $\mu=4$, which is less than the critical value for repetitive firing, and with the standard set of synaptic input parameters reported in column 1 of Table 1 in Destexhe et al. $(2001), \bar{g}_{e}=0.012 \mu \mathrm{S}, \bar{g}_{i}=0.057 \mu \mathrm{S}, \sigma_{e}=0.003, \sigma_{i}=0.0066$ but with $\tau_{e}=2, \tau_{i}=6$, and with initially resting conditions, a single sample path was generated as depicted in the top left part of Figure 8. A spike emerged which attained a maximum depolarization of about $97 \mathrm{mV}$ at approximately $8.5 \mathrm{~ms}$ with corresponding sample paths for $g_{e}$ and $g_{i}$ shown up to $20 \mathrm{~ms}$ in the top right part of Figure 8. Subsequently there are subthreshold fluctuations, labelled $V_{s}$ for $t \geq 40$ about a mean of $2.84 \mathrm{mV}$ depicted in the lower left part of the figure. A histogram of voltage values for $V_{s}$ from $t=40$ to $t=100$ and corresponding histograms of values of the excitatory and inhbitory conductances are also shown, being similar to those in Figure 2 of Destexhe et al. (2001).

Starting with the values of all components $V, n, m, h, g_{e}$ and $g_{i}$ at $t=40$ (beginning of $V_{s}$ ), three sets of simulations and moment equation calculations were performed with the results to $5 \mathrm{~ms}$ shown in Figure 9. There is good agreement between the means and variances determined by the two methods. 

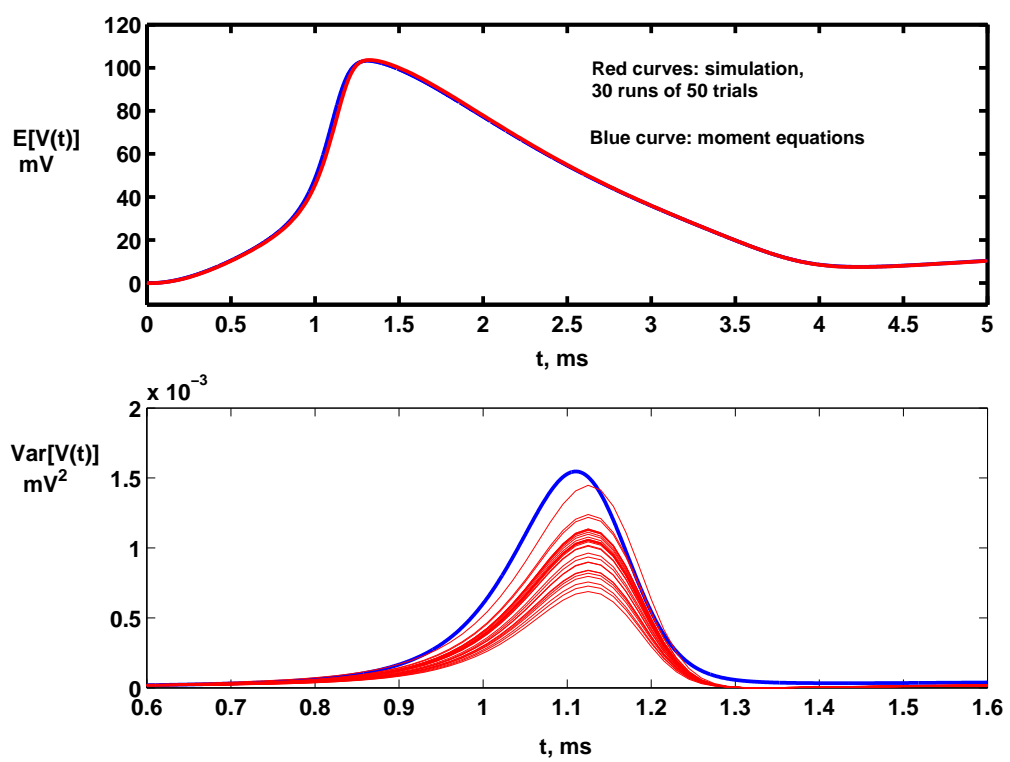

Figure 7: An example, with both excitation and inhibition, of the calculated mean and variance of the voltage $V(t)$ as functions of time of the voltage where there is fairly good agreement between results for simulation (red) and for moment equations (blue). The time scales are different for the mean and the variance. The principal synaptic input parameters are $\bar{g}_{e}=3, \bar{g}_{i}=1, \sigma_{e}=0.0003, \sigma_{i}=0.0002$. For remaining parameters see text. Results are given for 30 runs of 50 trials each. Note the variability in the curves for the variance by simulation (lower figure, red curves). The means for the two methods are practically indistinguishable.

Figure 10 illustrates further the occurrence of the occasional spike mode over a time period of $400 \mathrm{~ms}$. In all three records the inhibitory steady state conductance is the standard (Destexhe et al. (2001), Table 1, column 1) value of $\bar{g}_{i}=0.057$ and its noise amplitude is the standard value of $\sigma_{i}=0.0066$. In Figure $10 \mathrm{~A}$, there are no spikes with $\mu=4, \bar{g}_{e}$ at 4 times the standard value of 0.012 and with $\sigma_{e}=0.003$ which is the standard value. In the middle record of Figure 10B, there is slightly less additive depolarizing drive with $\mu=3.9$, less mean synaptic excitation with $\bar{g}_{e}$ at 3.6 times the standard value but with considerably greater noise amplitude of 3.2 times the standard value which is sufficient to induce occasional spiking. In Figure 10C, there is an initial singlet spike followed by two doublets with intra-doublet intervals of about $20 \mathrm{~ms}$. Here the parameters are all as in Figure 10A (no spikes) but the excitatory noise level is 3.25 times the standard value. With occasional spikes it is clear that the moment method will not be suitable because the condition of a symmetric distribution of component values is not met.

\subsubsection{Input parameters of Destexhe et al. (2001)}

As pointed out in the model description, Destexhe et al. (2001) studied a point HH model augmented with an M-type potassium current and random excitatory and inhibitory synaptic inputs. Apart from the M-type potassium current their model has the same structure 

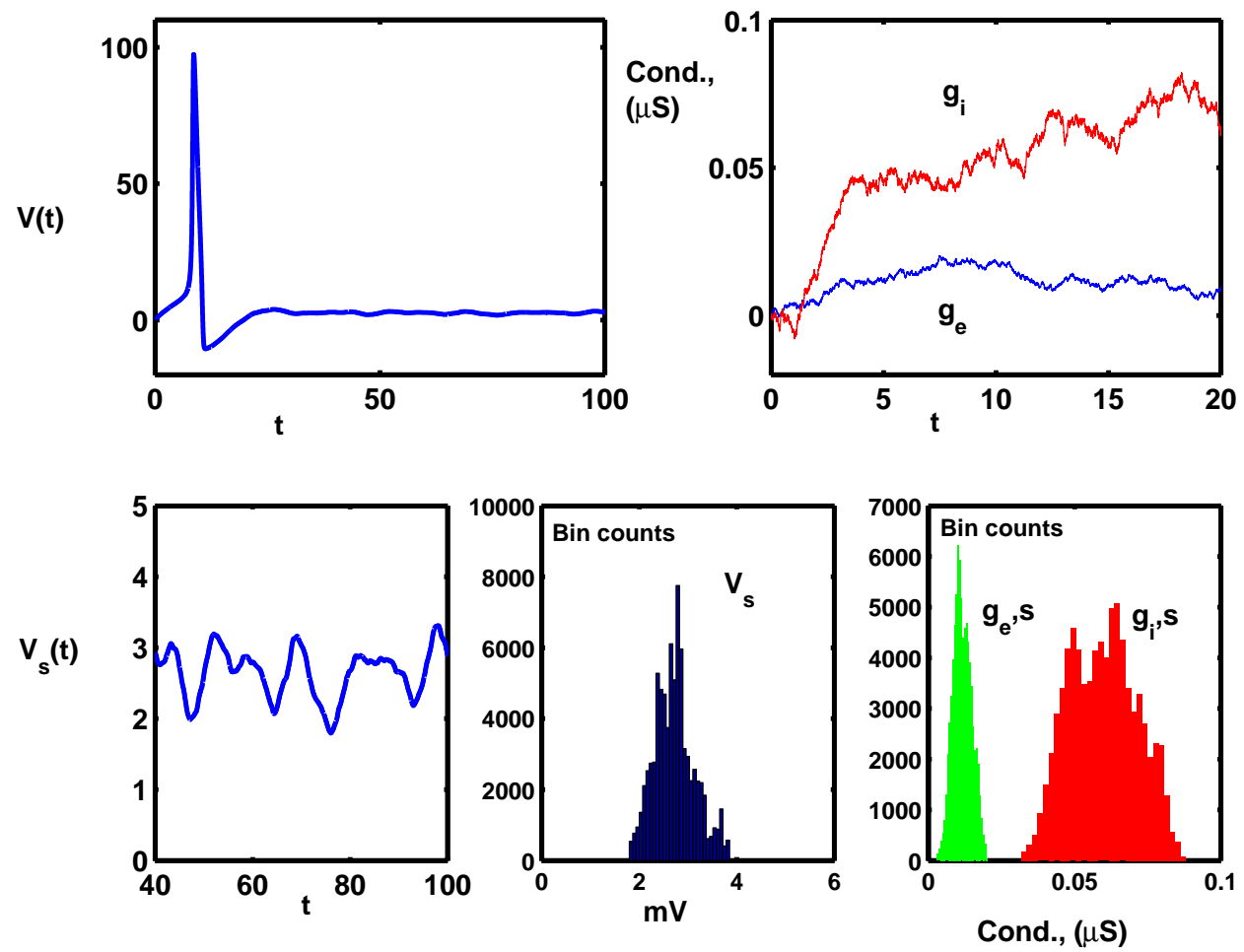

Figure 8: In the top left figure is shown a spike followed by fluctuations in a steady state up to $100 \mathrm{~ms}$. In the top right are shown the sample paths of $g_{e}$ and $g_{i}$ for the first $20 \mathrm{~ms}$. After $t=40$ the voltage fluctuations are designated $V_{s}$ with sample path and histogram of values shown in the first two bottom figures. In the bottom right figure are the histograms of $g_{e}$ and $g_{i}$ during the period from 40 to $100 \mathrm{~ms}$. The latter are comparable to those in Figures 2 and 3 of Destexhe et al. (2001). For parameter values, see text.
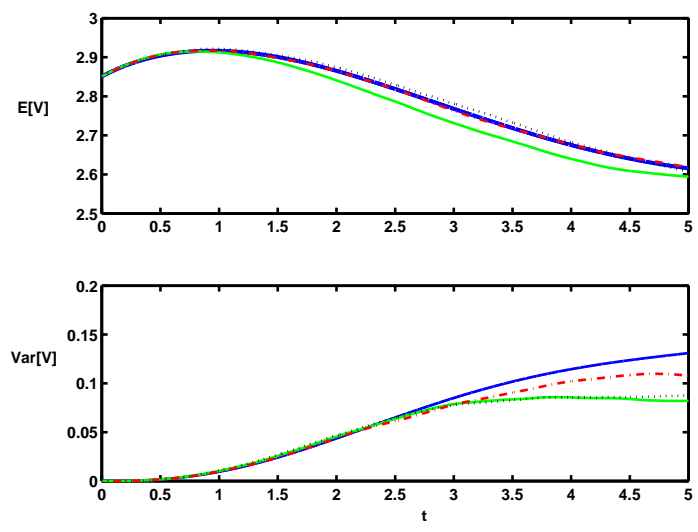

Figure 9: The mean and variance for the subthreshold voltage fluctuations of the previous figure calculated by the MEM (blue curves) and 3 sets of simulations (red, black and green curves). 

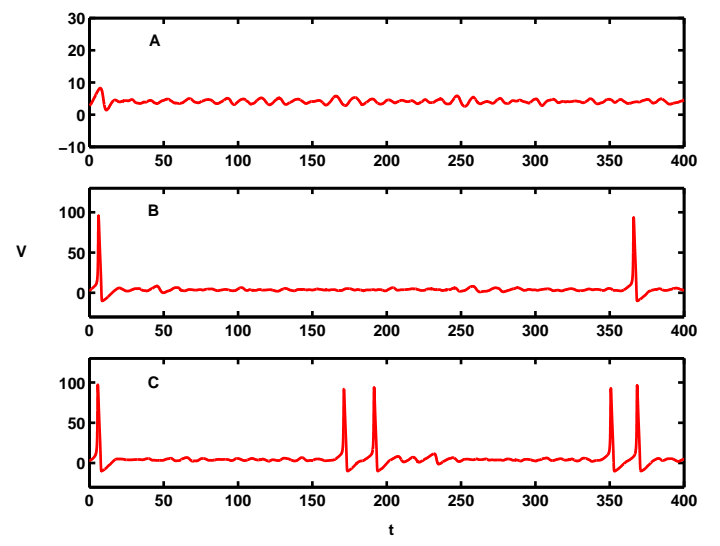

Figure 10: Sample paths of duration $400 \mathrm{~ms}$ with added current of strength $\mu$ and synaptic excitation and inhibition. A. Only subthreshold fluctuations occur for this period with $\mu=4, \bar{g}_{e}=0.048$ and $\sigma_{e}=0.003$. B. Occasional spike mode in which singlet spikes occur. Here $\mu=3.9, \bar{g}_{e}=0.0432$ and $\sigma_{e}=0.0096$. C. Occasional spike mode in which spikes occur, sometimes in pairs. Here $\mu=4, \bar{g}_{e}=0.048$ and $\sigma_{e}=0.0098$. Remaining parameters held fixed - see text.

as the one employed here.

It was of interest to see how results for the MEM compared with those for simulation, despite the lack of knowledge of the complete set of parameters for the transient sodium and delayed rectifier potassium current as well as the omission of the M-type current. We use data in column 1 of Table 1 of Destexhe et al. (2001) on properties of a layer 6 pyramidal cell (P-cell) of cat neocortex. A resting potential given in the text was stated to be $-80 \mathrm{mV}$.

The P-cell model parameters are as follows. In Equ. (11) we need $C$ the whole cell capacitance, which based on the given membrane area of $34,636 \mu \mathrm{m}^{2}$ is $0.34636 \mathrm{nF}$. If conductances are in $\mu \mathrm{S}$, and voltages are in $\mathrm{mV}$, then currents are in $\mathrm{nA}$. The equilibrium excitatory and inhibitory conductances for the whole cell are given as $\bar{g}_{e}=0.012 \mu \mathrm{S}, \bar{g}_{i}=$ $0.057 \mu \mathrm{S}$ with reversal potentials of $V_{e}=80 \mathrm{mV}$ and $V_{i}=5 \mathrm{mV}$ relative to a resting value of $V_{R}=0 \mathrm{mV}$. The leak conductance, using the value in Destexhe et al. (2001) is $g_{L}=0.01559 \mu \mathrm{S}$, which is about 6 times less than the value obtained if the standard $\mathrm{HH}$ value of $0.3 \mathrm{mS} / \mathrm{cm}^{2}$ is employed. For the values of $\bar{g}_{K}$ and $\bar{g}_{N a}$, we use the data of Paré et al. (1998) to obtain $3.4636 \mu \mathrm{S}$ and $2.4245 \mu \mathrm{S}$, respectively.

Results of a simulation for the P-cell model including synaptic input and with $\mathrm{HH}$ activation and inactivation dynamics and no added current are shown in Figure 11. The records have a duration of $100 \mathrm{~ms}$ during which the voltage, shown in the top left figure, fluctuates about a mean of $0.17 \mathrm{mV}$ (above rest) with a standard deviation of $1.6 \mathrm{mV}$. The distribution of $V$ is indicated by a histogram in the top right part of Figure 11. The lower two figures show the time courses of the excitatory and inhibitory conductances, $g_{e}$ and $g_{i}$. There are no spikes as the fluctuations do not take $V$ to threshold values. The mean in Destexhe et al. (2001) is much higher so that spikes do arise occasionally - called the occasional spike mode as mentioned above. The values of $g_{e}$ in Figure 11 are between about 0.001 and $0.023 \mu \mathrm{S}$; those of $g_{i}$ are between $-0.02 \mu \mathrm{S}$ and a maximum of about $0.09 \mu \mathrm{S}$. That 
the conductance may become slightly negative is a minor deficiency of the model equations, being due to the fact that the conductances are unrestricted OUPs. (This was allowed for in Tuckwell et al., 2009). Overall, the conductance fluctuations are comparable with those in Destexhe et al. (2001), being governed by the same stochastic differential equations.
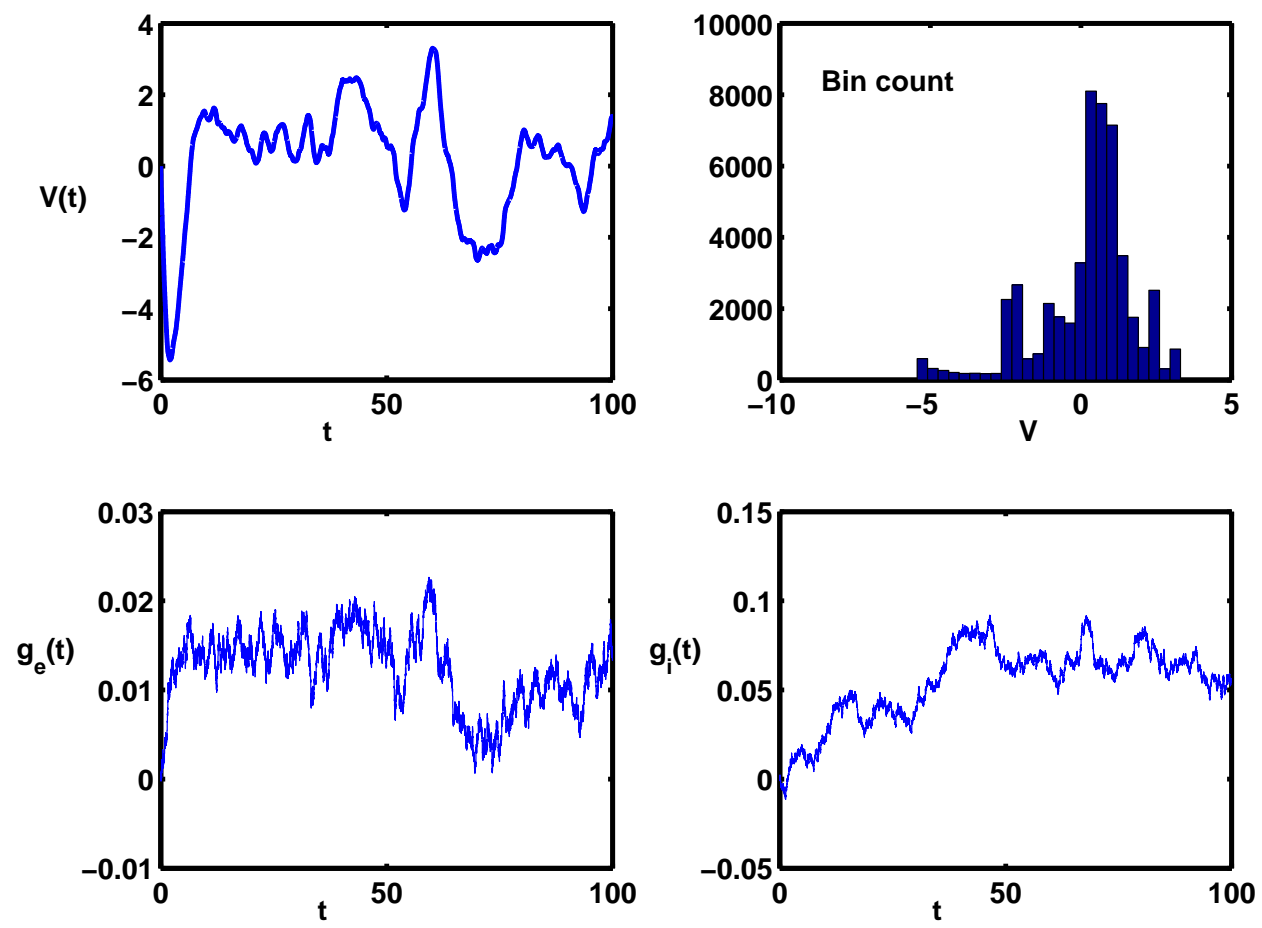

Figure 11: Properties of one sample path for the P-cell with excitatory and inhibitory synaptic input. The top left figure shows $V$ in $\mathrm{mV}$ versus $t$ in $\mathrm{ms}$ and the top right gives a histogram of the values of $V$ over the $100 \mathrm{~ms}$ period. In the lower two figures are the corresponding sample paths for the excitatory and inhibitory conductances. For parameters see text.

The P-cell model with $\mathrm{HH}$ activations and inactivation can be made to fire by either introducing an additive depolarizing current $\mu$ or by increasing the ranges of the fluctuations of the synaptic conductances. Figure 12 shows two such sets of results where results for simulation compare favorably with those obtained by solving the moment differential equations. In both cases there is an added depolarizing current $\mu=10 \mu \mathrm{A} / \mathrm{cm}^{2}$. For Figure $12 \mathrm{~A}$, the synaptic steady state conductances are $\bar{g}_{e}=1.2, \bar{g}_{i}=5.7$ with corresponding standard deviation parameters $\sigma_{e}=0.003$ and $\sigma_{i}=0.0066$ as above. After a broad small amplitude spike, a steady state is attained at a depolarized level with no further spikes. The agreement of the MEM and simulation for the mean and variance of $V$ is very good throughout the spike and immediately afterwards. The variance rises and falls during the rising and falling phases of the spike. In Figure 12B, the steady state synaptic conductances have the smaller values $\bar{g}_{e}=0.12, \bar{g}_{i}=0.57$ with larger corresponding standard deviation parameters $\sigma_{e}=0.03$ and $\sigma_{i}=0.066$. Again a small ampltude broad spike forms after which a fluctuating steady state is attained. Interesting is the fact that in this case the 
variance has two maxima: it rises on the leading edge of the spike, decreases around the peak, rises again until about half-way down the falling edge and then declines to a steady state value. This behavior of the variance is confirmed by the calculation by MEM.
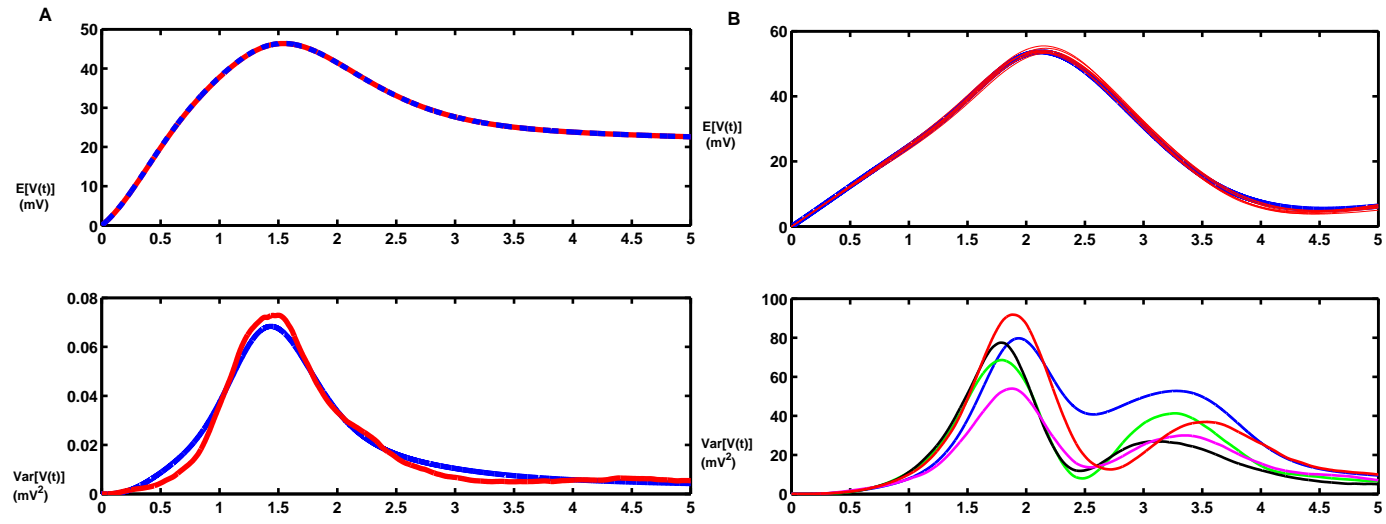

Figure 12: A. An example for the P-cell model with an added depolarizing current and strong synaptic excitation and inhibition such that threshold for spiking is exceeded. Here there is very good agreement between the mean and variance of $V(t)$ determined by simulation (red curves) and by the MEM (blue curves). B. A second example for the P-cell model with the same depolarizing current as in A but with weaker but noisier synaptic excitation and inhibition. There are 4 sets of simulations with the same number of trials. There is broad agreement between the variance versus time curves from simulation (various colors) and the result from MEM (blue curve). Note the secondary maximum in the variance. For both $\mathrm{A}$ and $\mathrm{B}$ the means for the two methods are practically indistinguishable. For parameter values see text.

\section{Discussion and conclusions}

The Hodgkin-Huxley (1952) mathematical model for voltage and current responses in squid axon has formed a cornerstone for describing the dynamics of neuronal subthreshold and spiking activity in a large number of types of neurons in many parts of the nervous system in diverse species. Whereas the original model involved only three types of ionic current, being leak, potassium and sodium, models of most neurons have been found to require several types of sodium, potassium and calcium currents, each of which has a distinct role in determining a cell's electrophysiological properties. The HH system itself is an important starting point in the analysis of neuronal behavior and is used in the present paper to examine the effects of random synaptic activity on repetitive spiking. Many of the findings are expected to carry over to more complex models.

The present article contains an extension of some previous studies of the effects of noise in space-clamped HH models. Firstly, the results of the inclusion of additive white noise and excitatory synaptic noise represented by OUPs, on periodic spiking (Tuckwell et al., 2009). Secondly, the use of a system of deterministic differential equations to find 
approximately the first and second-order moments of the $\mathrm{HH}$ variables $V, n, m, h$ (Rodriguez and Tuckwell, 2000; Tuckwell and Jost, 2009). In the first study it was found that at input signals just greater than critical values for repetitive (limit cycle) spiking, weak noise could cause a substantial decrease in firing rate and that a minimum in firing rate occurred as the noise level increased from zero. In the second type of study, the system of ordinary differential equations for the approximate moments was solved and the results compared with simulations.

The model employed here includes synaptic input of the type supported by experimental analysis and modeling of the voltage response of cat and rat neocortical neurons (Destexhe et al, 2001; Fellous et al., 2003). The spiking observed in those experiments was occasional (Calvin, 1975) and the inputs were described as fast with small amplitude so that they could be represented by continuous random processes (OUPs). We have investigated the effects of these types of excitatory and inhibitory random inputs on repetitive spiking in the $\mathrm{HH}$ model. However, it is likely that similar qualitative results would obtain if the random synaptic inputs were purely additive.

The effect of weak random excitatory synaptic input, investigated in Section 4.1.2, was to inhibit spiking induced by a purely excitatory current near the threshold for repetitive firing, confirming results in Section IV of Tuckwell et al. (2009). Section 4.1 .3 contained results for the effects of noisy synaptic input on repetitive spiking induced by the simultaneous application of steady excitatory and steady inhibitory currents. Here it was demonstrated (Figure 4) that weak noise in either the excitatory or inhibitory input process or in both could strongly inhibit spiking. Thus a small amount of noise in any synaptic input channel can cause a dimunition of repetitive firing near threshold. Of considerable interest were the findings that with both steady excitatory and inhibitory current, with no noise in the excitatory input, increasing the noise only in the inhibitory component from zero, gave rise to a minimum in the firing rate as in the phenomenon of inverse stochastic resonance previously demonstrated for the $\mathrm{HH}$ model with additive noise and synaptic excitation only (Figure 5).

The differential equations for the approximate first and second order moments of the model were derived in Section 3. The system consists of six equations for the means, six for the variances and fifteen for the covariances giving 27 differential equations in total. These were solved numerically with a Runge-Kutta routine and the results compared with those obtained by simulation. When the level of excitation is too weak to evoke regular firing, the assumptions for the validity of the moment method are not satisfied and agreement with simulation is generally poor. However, when the firing rate was larger, either with stronger excitatory synaptic input or with an additional added depolarizing current, and when the noise was of small amplitude, then in a few examples good agreement was found between the two methods over small time intervals, as in the Fitzhugh-Nagumo system with additive noise (Rodriguez and Tuckwell, 1996).

\section{Acknowledgements}

We appreciate helpful correspondence with Dr Alain Destexhe at CNRS, Gif-sur-Yvette, France. The work is part of the Dynamical Systems Interdisciplinary Network, University 
of Copenhagen.

\section{Appendix: $\mathrm{HH}$ parameters, coefficients and their derivatives}

\section{Coefficients and their 1st and 2nd derivatives}

Recalling that here $V$ is depolarization and not membrane potential (cf the original forms in $\mathrm{HH}(1952)$ ), the coefficients in the auxiliary equations are the following standard ones.

$$
\begin{aligned}
\alpha_{n}(V)=10-V \varnothing 100\left[e^{(10-V) / 10}-1\right], & \beta_{n}(V)=1 \varnothing 8 e^{-V / 80}, \\
\alpha_{m}(V)=25-V \varnothing 10\left[e^{(25-V) / 10}-1\right], & \beta_{m}(V)=4 e^{-V / 18}, \\
\alpha_{h}(V)=7 \varnothing 100 e^{-V / 20}, & \beta_{h}(V)=1 \varnothing e^{(30-V) / 10}+1 .
\end{aligned}
$$

In the moment equations we require their first and second order derivatives. The latter are

$$
\begin{aligned}
\alpha_{n}^{\prime}(V)=\frac{10-V e^{1-\frac{V}{10}}}{1000\left(e^{1-\frac{V}{10}}-1\right)^{2}}, & \beta_{n}^{\prime}(V)=-\frac{1}{640} e^{-V / 80}, \\
\alpha_{m}^{\prime}(V)=\frac{10+e^{\frac{25-V}{10}}(15-V)}{100\left(e^{\frac{25-V}{10}}-1\right)^{2}}, & \beta_{m}^{\prime}(V)=-\frac{2}{9} e^{-V / 18}, \\
\alpha_{h}^{\prime}(V)=-\frac{7}{2000} e^{-V / 20}, & \beta_{h}^{\prime}(V)=\frac{e^{\frac{30-V}{10}}}{10\left(e^{\frac{30-V}{10}}+1\right)^{2}} .
\end{aligned}
$$

The corresponding second derivatives are found to be

$$
\begin{aligned}
\alpha_{n}^{\prime \prime}(V)=\frac{2 \beta[(10-\beta V)-5(\beta e-1)(1-V / 10)]}{10^{4}(\beta e-1)^{3}}, & \beta_{n}^{\prime \prime}(V)=\frac{1}{51200} e^{-V / 80}, \\
\alpha_{m}^{\prime \prime}(V)=\frac{2 \alpha(\alpha(15-V)+10)-10 e^{\frac{-V}{10}}(25-V)(\alpha-1)}{1000(\alpha-1)^{3}}, & \beta_{m}^{\prime \prime}(V)=\frac{1}{81} e^{-V / 18}, \\
\alpha_{h}^{\prime \prime}(V)=\frac{7}{40000} e^{-V / 20}, & \beta_{h}^{\prime \prime}(V)=\frac{\gamma}{100}\left(\frac{1}{(1+\gamma)^{2}}+\gamma\right),
\end{aligned}
$$

where

$$
\alpha=e^{\frac{25-V}{10}}, \quad \beta=e^{-\frac{V}{10}}, \quad \gamma=e^{\frac{30-V}{10}} .
$$

\section{Standard parameters and initial values}

The values of the $\mathrm{HH}$ parameters employed in the present model are the standard ones: $C=1, \bar{g}_{K}=36, \bar{g}_{N} a=120, g_{L}=0.3, V_{K}=-12, V_{N a}=115$ and $V_{L}=10$. The initial (resting) values are $V(0)=0$,

$$
m(0)=\frac{\alpha_{m}(0)}{\alpha_{m}(0)+\beta_{m}(0)}, \quad h(0)=\frac{\alpha_{h}(0)}{\alpha_{h}(0)+\beta_{h}(0)}, \quad n(0)=\frac{\alpha_{n}(0)}{\alpha_{n}(0)+\beta_{n}(0)} .
$$

The numerical values of the last three quantities are $0.0529,0.5961$ and 0.3177 , respectively. 


\section{References}

Austin, T.D., 2008. The emergence of the deterministic Hodgkin-Huxley equations as a limit from the underlying stochastic ion-channel mechanism. The Annals of Applied Probability 18, 1279-1325.

Bachar, M., Batzel, J.J. and Ditlevsen, S., Eds., 2013. Stochastic Biomathematical Models: with Applications to Neuronal Modeling. Lecture Notes in Mathematics 2058. Springer, Berlin.

Bashkirtseva, I., Neiman, A. B., Ryashko, L. (2015). Stochastic sensitivity analysis of noiseinduced suppression of firing and giant variability of spiking in a Hodgkin-Huxley neuron model. Physical Review E, 91(5), 052920.

Berglund, N., Kuehn, C., 2015. Regularity structures and renormalisation of FitzHughNagumo SPDEs in three space dimensions. arXiv preprint arXiv:1504.02953.

Berg, R.W. and Ditlevsen, S., 2013. Synaptic inhibition and excitation estimated via the time constant of membrane potential fluctuations. Journal of Neurophysiology, 110: 1021-1034.

Blair, E.A., Erlanger, J., 1932. Responses of axons to brief shocks. Experimental Biology and Medicine 29, 926-927.

Brink, F., Bronk, D. W., Larrabee, M. G.,1946. Chemical excitation of nerve. Ann. N.Y. A cad. Sci. 47, 457-85.

Burns, B. D., Webb, A. C.,1976. The spontaneous activity of neurones in the cat's cerebral cortex. Proc. Roy. Soc. Lond. B. 194, 211-23.

Calvin, W.H., 1975. Generation of spike trains in CNS neurons. Brain Res. 84, 1-22.

Clay, J.R., 2005. Axonal excitability revisited. Progress in Biophysics and Molecular Biology 88, 59-90.

Colquhoun, D., Hawkes, A.G., 1981. On the stochastic properties of single ion channels. Proceedings of the Royal Society of London B: Biological Sciences 211, 205-235.

Deco, G. and Marti, D., 2007. Extended method of moments for deterministic analysis of stochastic multistable neurodynamical systems. Physical Review 75, 031913.

Deco, G., Ponce-Alvarez, A., Mantini, D. et al., 2013. Resting-state functional connectivity emerges from structurally and dynamically shaped slow linear fluctuations. J. Neurosci. 33, 11239-11252.

Destexhe, A., Rudolph, M. , Fellous, J-M., Sejnowski, T.J., 2001. Fluctuating synaptic conductances recreate in vivo-like activity in neocortical neurons. Neuroscience 107, $13-24$.

Ditlevsen, S., Lansky, P., 2005. Estimation of the input parameters in the OrnsteinUhlenbeck neuronal model. Physical Review E 71,011907.

Ditlevsen, S., 2007. A result on the first-passage time of an Ornstein-Uhlenbeck process. Statistics and Probability Letters 77, 1744-1749.

Fellous, J-M., Rudolph, M., Destexhe, A., Sejnowski, T.J., 2003. Synaptic background noise controls the input/ output characteristics of single cells in an in vitro model of in vivo activity. Neuroscience 122, 811-829.

Finke, C., Vollmer, J., Postnova, S. and Braun, H.A., 2008. Propagation effects of current and conductance noise in a model neuron with subthreshold oscillations. Mathematical Biosciences 214, 109-121. 
Franović, I., Todorović, K., Vasović, N.,Burić, N., 2013. Mean-field approximation of two coupled populations of excitable units. Phys. Rev. E 87, 012922.

Gerstein, G. L., Kiang, N. Y.-S., 1960. An approach to the quantitative analysis of electrophysiological data from single neurons. Biophys. J. 1, 15-28.

Gihman, I. I. and Skorohod, A.V. (1972). Stochastic Differential Equations. Springer, Berlin.

Guo, D., 2011. Inhibition of rhythmic spiking by colored noise in neural systems. Cognitive neurodynamics 5, 293-300.

Hasegawa, H., 2009. Population rate codes carried by mean, fluctuation and synchrony of neuronal firings. Physica A 388, 499-513.

Hasegawa, Y., 2015. Variational superposed Gaussian approximation for time-dependent solutions of Langevin equations. Physical Review E 91, 042912.

Hillenbrand, U., 2002. Subthreshold dynamics of the neural membrane potential driven by stochastic synaptic input. Physical Review E, 66, 021909.

Hodgkin, A.L. and Huxley, A.F. (1952) A quantitative description of membrane current and its application to conduction and excitation in nerve. J. Physiol. (Lond.), 117 500-544.

Horikawa, Y., 1991. Noise effects on spike propagation in the stochastic Hodgkin-Huxley models. Biological cybernetics 66,19-25.

Lecar, H., Nossal, R., 1971. Theory of threshold fluctuations in nerves: I. Relationships between electrical noise and fluctuations in axon firing. Biophysical Journal 11,10481067.

Li, Y., Schmid, G., Hänggi, P., Schimansky-Geier, L., 2010. Spontaneous spiking in an autaptic Hodgkin-Huxley setup. Physical Review E 82, 061907.

Marreiros, A.C., Kiebel, S.J., Daunizeau, J. et al., 2009. Population dynamics under the Laplace assumption. NeuroImage 44, 701-714.

Monnier, A.M. and Jasper, H.H., 1932. Recherches de la relation entre les potentiels d'action lmentaires et la chronaxie de subordination. Nouvelle dmonstration du fonctionnement par Tout ou Rien de la fibre nerveuse. C.R. Soc. Biol. 110,, 547-549.

Paré, D, Lang, E.J., Destexhe, A., 1998. Inhibitory control of somatodendritic interactions underlying action potentials in neocortical pyramidal neurons in vivo: an intracellular and computational study. Neuroscience 84, 377-402.

Pecher, C., 1936. Etude statistique des variations spontanées de l'excitabilité d'une fibre nerveuse. C.R. Soc. Biol. 122, 87-91.

Pecher, C., 1937. Fluctuations indépendantes de l'excitabilité de deux fibres d'une même nerf. C.R. Soc. Biol., Paris. 124, 839-842.

Rodriguez, R., Tuckwell, H.C., 1996. Statistical properties of stochastic nonlinear dynamical models of single spiking neurons and neural network. Phys. Rev. E 54, 5585-5589.

Rodriguez, R., Tuckwell, H.C., 1998. Noisy spiking neurons and networks: useful approximations for firing probabilities and global behavior. BioSystems 48, 187-194.

Rodriguez, R., Tuckwell, H.C., 2000. A dynamical system for the approximate moments of nonlinear stochastic models of spiking neurons and networks. Mathematical and Computer Modelling 31, 175-180.

Sauer, M., Stannat, W., 2016. Reliability of signal transmission in stochastic nerve axon equations. Journal of Computational Neuroscience. To appear. arXiv preprint 
arXiv:1502.04295.

Sokolowski, T. R., Tkačik, G., 2015. Optimizing information flow in small genetic networks. IV. Spatial coupling. arXiv preprint arXiv:1501.04015.

Stannat, W., 2013. Stability of travelling waves in stochastic Nagumo equations. arXiv preprint arXiv:1301.6378.

Tuckwell, H.C., 1987. Diffusion approximations to channel noise. Journal of Theoretical Biology 127, 427-438.

Tuckwell, H.C., 2005. Spike trains in a stochastic Hodgkin-Huxley system. BioSystems 80, $25-36$.

Tuckwell, H.C., 2008. Analytical and simulation results for the stochastic spatial FitzhughNagumo model neuron. Neural Computation 20, 3003-3033.

Tuckwell, H.C., Jost, J., 2009. Moment analysis of the Hodgkin-Huxley system with additive noise. Physica A 388, 4115-4125.

Tuckwell, H. C., Jost, J. (2012). Analysis of inverse stochastic resonance and the long-term firing of HodgkinHuxley neurons with Gaussian white noise. Physica A: Statistical Mechanics and its Applications, 391(22), 5311-5325.

Tuckwell, H.C., Jost, J. and Gutkin, B.S. (2009). Inhibition and modulation of rhythmic neuronal spiking by noise. Phys. Rev. E 80, 031907.

Tuckwell, H.C. and Rodriguez, R. (1998). Analytical and simulation results for stochastic Fitzhugh-Nagumo neurons and neural networks. J. Computational Neuroscience 5, 91-113.

Verveen, A. A., 1960. On the fluctuation of threshold of the nerve fibre. In: Structure and Function of the Cerebral Cortex, pp 282-288. Eds., Tower, D.P., Schad, J.P.). Elsevier, Amsterdam.

Wenning, G., Hoch, T., Obermayer, K., 2005. Detection of pulses in a colored noise setting. Physical Review E 71, 021902.

White, J.A., Rubinstein, J.T., Kay, A.R., 2000. Channel noise in neurons. Trends in Neurosciences 23, 131-137.

Yu, Y., Shu, Y., McCormick, D.A., 2008. Cortical action potential backpropagation explains spike threshold variability and rapid-onset kinetics. J. Neurosci. 28., 7260-7272. 